\title{
Editorial
}

\section{Stage IV melanoma}

\author{
Paul Porensky, M.D., and E. Antonio Chiocca, M.D., Ph.D.
}

Department of Neurological Surgery, College of Medicine, The Ohio State University, Columbus, Ohio

Stage IV melanoma is characterized by a propensity for the development of cranial metastatic disease and the markedly worse prognosis that such CNS involvement carries. Indeed, various reviews cite a $10 \%-40 \%$ incidence of known brain metastases in patients with systemic melanoma, with up to $75 \%$ of cases having autopsy-proven brain involvement. ${ }^{1,6}$ Melanoma has the highest frequency of cerebral spread among all primary neoplasms. Coupled with this CNS tropism is the resulting morbidity and mortality incremental to the burden of systemic disease. For patients with melanoma brain metastases, neurological decline is frequently the cause of death.

Untreated disease carries the grim prognosis of death within 1 month. Treatment modifiers, including steroids, surgery, whole-brain radiation therapy (WBRT), stereotactic radiosurgery (SRS), and chemotherapy, can extend median survival to a little less than 1 year when delivered in various combinations. ${ }^{6}$ In a morbid rendition of whacka-mole, these interventions often quell, for a time, CNS advancement only to allow the systemic disease to advance beyond treatment. ${ }^{2,5}$ More recently there has been notable interest in developing immune-modulating therapies as part of the melanoma armamentarium. The concept encompasses such diverse forays as antitumor vaccines and immune activators with the goal of harnessing the power of the patient's own immune system. Measurable success in the setting of Stage IV melanoma without CNS spread has been documented, ${ }^{3}$ whereas scattered and only smallcohort studies have investigated disease with cranial involvement. $^{6}$

The study by Knisely et al. published in this issue of the Journal of Neurosurgery adds an intriguing chapter to immune-modulating therapy in the setting of Stage IV melanoma with brain metastases. ${ }^{4}$ The authors undertook a retrospective chart review for disease modifiers in $77 \mathrm{pa}-$ tients who had undergone SRS between 2002 and 2010. Twenty-seven patients who also received ipilimumab immunotherapy were identified, and both overall and 2-year median survivals were compared with survival in 50 patients in the unmatched control group. The SRS with ipilimumab group yielded an overall median survival of 21.3 months compared with 4.9 months in patients with SRS but without ipilimumab. Two-year survival was also starkly improved from $19.7 \%$ to $47 \%$. Multivariate analysis confirmed these results after controlling for some known outcome modifiers, including the Diagnosis-Specific Graded Prognostic Assessment, a validated scale incorporating both performance status and quantity of cerebral metastases.

Ipilimumab is a fully human antibody that is designed to block the negative T-cell signaling receptor cytotoxic $\mathrm{T}$ lymphocyte-associated antigen 4 (CTLA-4) ${ }^{8}$ This receptor is in effect a checkpoint for continued T-cell activation; receptor triggering promotes a return to homeostasis and avoidance of an overwhelming and detrimental immune response. Steric block by ipilimumab enhances the time course and intensity of the immune response and thus circumvents immune tolerance and purportedly enhances control of systemic tumor. ${ }^{3,8}$ This strategy is particularly suitable to melanoma, one of the more immunogenic solid tumors. ${ }^{6}$ One large Phase III trial for Stage IV melanoma without cranial disease showed improved survival (10.0 vs 6.4 months) with ipilimumab therapy. ${ }^{3}$ Therefore, particularly exciting in the Knisely et al. review is the suggestion for a first-of-its-kind therapy that offers substantive disease modulation for both cranial and systemic components of Stage IV melanoma. The large relative increases in survival, coupled with the authors' finding that there was not a statistically significant increase in the need for salvage WBRT or SRS that might be expected with prolonged survival, offer more than a glimmer of hope for a meaningful intervention in a disease that has been historically resistant to treatment.

The study does have limitations, most notably in the retrospective quality of data acquisition and the accompanying biases that permeate such reviews, including referral and reporting biases, the lack of appropriately matched controls, and a single-academic-center experience that precludes extrapolation to the general population. Specifically, all study patients needed to have a good performance status even for SRS consideration. Moreover, all patients receiving ipilimumab were functional without assistance in their activities of daily living based on an Eastern Cooperative Oncology Group performance score $\leq 2$, although performance criteria for the control group were not defined and perhaps were more variable; the ipilimumab group was also statistically younger (median 53 vs 59 years). Importantly, some key modifiers of survival were not reported, including pretreatment levels of lactate dehydrogenase, hemorrhage within the metastatic tumor bed, recursive partitioning analysis class, and status of systemic disease at treatment onset. ${ }^{2,5,7}$ One last important unknown is any de- 
finitive inclusion and exclusion criteria qualifying a patient for ipilimumab therapy.

Notwithstanding these limitations, all inherent to a retrospective review, the impressive survival differences argue for aggressive prospective evaluation of ipilimumab in patients with Stage IV melanoma with cerebral metastases. These results should validate the concept of immune therapy efficacy even in the presence of CNS involvement, thereby opening the door for inclusion of such patients in clinical trials of future immune therapeutics.

(http://thejns.org/doi/abs/10.3171/2012.2.JNS1288)

\section{Disclosure}

The authors report no conflict of interest.

\section{References}

1. Bafaloukos D, Gogas H: The treatment of brain metastases in melanoma patients. Cancer Treat Rev 30:515-520, 2004

2. Eigentler TK, Figl A, Krex D, Mohr P, Mauch C, Rass K, et al: Number of metastases, serum lactate dehydrogenase level, and type of treatment are prognostic factors in patients with brain metastases of malignant melanoma. Cancer 117:16971703, 2011

3. Hodi FS, O'Day SJ, McDermott DF, Weber RW, Sosman JA, Haanen JB, et al: Improved survival with ipilimumab in patients with metastatic melanoma. N Engl J Med 363:711-723, 2010

4. Knisely JPS, Yu JB, Flanigan J, Sznol M, Kluger HM, Chiang VLS: Radiosurgery for melanoma brain metastases in the ipilimumab era and the possibility of longer survival. Clinical article. J Neurosurg [epub ahead of print June 15, 2012. DOI: 10.3171/2012.5.JNS111929]

5. Liew DN, Kano H, Kondziolka D, Mathieu D, Niranjan A, Flickinger JC, et al: Outcome predictors of Gamma Knife surgery for melanoma brain metastases. Clinical article. J Neurosurg 114:769-779, 2011

6. Sloan AE, Nock CJ, Einstein DB: Diagnosis and treatment of melanoma brain metastasis: a literature review. Cancer Control 16:248-255, 2009

7. Staudt M, Lasithiotakis K, Leiter U, Meier F, Eigentler T, Bamberg M, et al: Determinants of survival in patients with brain metastases from cutaneous melanoma. Br J Cancer 102:1213-1218, 2010

8. Tarhini AA, Iqbal F: CTLA-4 blockade: therapeutic potential in cancer treatments. Onco Targets Ther 24:15-25, 2010

\section{Response}

Jonathan P. S. Knisely, M.D., ${ }^{1}$ James B. Yu, M.D., ${ }^{3,4}$ Jaclyn Flanigan, M.D., ,,3 Mario Sznol, M.D., ${ }^{2,3}$ Harriet M. Kluger, M.D., ${ }^{2,3}$ and Veronica L. S. Chiang, M.D.,

${ }^{1}$ Department of Radiation Medicine, Hofstra University North Shore-LIJ School of Medicine, Hofstra University, Manhasset, New York; ${ }^{2}$ Department of Internal Medicine, Section of Medical
Oncology; Departments of ${ }^{4}$ Therapeutic Radiology and ${ }^{5}$ Neurosurgery, Yale University School of Medicine; and ${ }^{3}$ Yale Cancer Center, New Haven, Connecticut

We thank Drs. Porensky and Chiocca for their insightful commentary. We are heartened by the prospect of focal interventions for brain metastases providing an opportunity to improve survival for patients with Stage IV melanoma, now that effective immune-mediated systemic therapy is available.

Several important points in their editorial deserve reiteration: The retrospective nature of our study should highlight the potential for variation in patient management decisions across the time period that patients were treated. In addition, the possibility that there were substantial, unrecorded differences between the cohorts in terms of important prognostic indicators cannot be ignored.

Although we did not provide the Radiation Therapy Oncology Group (RTOG) recursive partitioning analysis (RPA) score, which has been found by some investigators to have prognostic significance, we did provide the melanoma Diagnosis-Specific Graded Prognostic Assessment (DS-GPA) scores. The melanoma DS-GPA has never been directly compared with the RTOG RPA in assessing prognoses in patients with melanoma, but its predecessor was believed to be as prognostic as the RPA, although less subjective. ${ }^{1}$ In the multiinstitutional cohort of 483 patients from which the DS-GPA for melanoma was derived, the only components of the GPA that retained prognostic significance in an RPA and multivariable Cox regression analysis were the number of brain metastases and the performance status, making it even easier to apply and more specific than its predecessor. ${ }^{2}$

We also agree with Porensky and Chiocca that given our results, aggressive prospective evaluation of ipilimumab in patients with Stage IV melanoma and brain metastases is warranted. Finally, we believe that further work must be done to determine the effects of immune-mediated therapies on the CNS given the protracted survival that these patients may enjoy.

\section{References}

1. Sperduto PW, Berkey B, Gaspar LE, Mehta M, Currran W: A new prognostic index and comparison to three other indices for patients with brain metastases: an analysis of 1,960 patients in the RTOG database. Int J Radiat Oncol Biol Phys 70:510-514, 2008

2. Sperduto PW, Chao ST, Sneed PK, Luo X, Suh J, Roberge $\mathrm{D}$, et al: Diagnosis-specific prognostic factors, indexes, and treatment outcomes for patients with newly diagnosed brain metastases: a multi-institutional analysis of 4,259 patients. Int J Radiat Oncol Biol Phys 77:655-661, 2010

Please include this information when citing this paper: published online June 15, 2012; DOI: 10.3171/2012.2.JNS1288. 